\title{
Identification of differentially expressed genes and enriched pathways in lung cancer using bioinformatics analysis
}

\author{
TINGTING LONG ${ }^{1}$, ZIJING LIU ${ }^{2}$, XING ZHOU ${ }^{1}$, SHUANG YU $^{1}$, HUI TIAN ${ }^{1}$ and YIXI BAO ${ }^{1}$ \\ ${ }^{1}$ Department of Laboratory Medicine, The Second Affiliated Hospital of Chongqing Medical University, \\ Chongqing 400010; ${ }^{2}$ Department of Clinical Medicine, Xinjiang Medical University, Urumqi, Xinjiang 830054, P.R. China
}

Received December 27, 2017; Accepted October 16, 2018

DOI: $10.3892 / \mathrm{mmr} .2019 .9878$

\begin{abstract}
Lung cancer is the leading cause of cancer-associated mortality worldwide. The aim of the present study was to identify the differentially expressed genes (DEGs) and enriched pathways in lung cancer by bioinformatics analysis, and to provide potential targets for diagnosis and treatment. Valid microarray data of 31 pairs of lung cancer tissues and matched normal samples (GSE19804) were obtained from the Gene Expression Omnibus database. Significance analysis of the gene expression profile was used to identify DEGs between cancer tissues and normal tissues, and a total of 1,970 DEGs, which were significantly enriched in biological processes, were screened. Through the Gene Ontology function and Kyoto Encyclopedia of Genes and Genomes (KEGG) pathway enrichment analysis, 77 KEGG pathways associated with lung cancer were identified, among which the Toll-like receptor pathway was observed to be important. Protein-protein interaction network analysis extracted 1,770 nodes and 10,667 edges, and identified 10 genes with key roles in lung cancer with highest degrees, hub centrality and betweenness. Additionally, the module analysis of protein-protein interactions revealed that 'chemokine signaling pathway', 'cell cycle' and 'pathways in cancer' had a close association with lung cancer. In conclusion, the identified DEGs, particularly the hub genes, strengthen the understanding of the development
\end{abstract}

Correspondence to: Professor Yixi Bao, Department of Laboratory Medicine, The Second Affiliated Hospital of Chongqing Medical University, 76 Linjiang Road, Yuzhong, Chongqing 400010, P.R. China

E-mail: yixibao@163.com

Abbreviations: DEGs, differentially expressed genes; KEGG, Kyoto Encyclopedia of Genes and Genomes; PPI, protein-protein interaction; GEO, Gene Expression Omnibus; BP, biological processes; MF, molecular function; $\mathrm{CC}$, cellular component; DAVID, Database for Annotation, Visualization and Integrated Discovery; FC, fold-change; STRING, Search Tool for the Retrieval of Interacting Genes

Key words: differentially expressed genes, lung cancer, bioinformatics analysis and progression of lung cancer, and certain genes (including advanced glycosylation end-product specific receptor and epidermal growth factor receptor) may be used as candidate target molecules to diagnose, monitor and treat lung cancer.

\section{Introduction}

Lung cancer is one of the most frequent malignancies worldwide and is the most common cause of global cancer-associated mortality, with over a million succumbing each year (1). Global mortality from lung cancer increased from 3.5 million in 1990 to 4.2 million in 2015 (2) and it is estimated that there will be 2.1 million new lung cancer cases and 1.8 million deaths in 2018, representing close to 1 in 5 (18.4\%) cases of cancer-associated mortality (3). The five-year survival rate for lung cancer patients is very low. This may be attributed to the lack of effective therapeutic methods and the difficulty in early diagnosis for lung cancer (4). Lung cancer is considered to be a heterogeneous disease and a number of factors including genetic mutations, environmental factors and individual habits can contribute to cancer occurrence, progression and metastasis (5). A number of genes and cellular pathways have been reported to participate in these processes $(6,7)$. Thus, understanding the precise molecular mechanisms underlying lung cancer progression is important for the development of diagnostic and therapeutic strategies.

Microarray has increasingly become a promising tool in studying medical oncology (8). A previous study on gene expression profiling in cancer used microarray technology (9), but only a few of these studies have been conducted on lung cancer (10). In addition, comparative analysis of the differentially expressed genes (DEGs) remains relatively limited (10), and a reliable biomarker profile discriminating cancer from normal tissues remains to be identified. The expression changes of genes in the development and progression of lung cancer require further analysis. In addition, the interactions among the identified DEGs, particularly the important signaling pathways and the interaction networks, should be elucidated.

In the present study, original data (GSE19804) were downloaded from Gene Expression Omnibus (GEO; www. ncbi.nlm.nih.gov/geo), which is a hub for the archiving of microarray data and their retrieval. Following the elimination of mismatched chips, the DEGs between lung cancer tissues and normal tissues were identified by comparing gene 
expression profiles. Subsequently, the DEGs were screened using Gene-Spring software for Gene Ontology (GO) and pathway enrichment analysis. By investigating their hub nodes and modules using a protein-protein interaction (PPI) network, the present study aimed to further understand the molecular mechanisms for lung cancer development and to identify potential candidate biomarkers for diagnosis, therapeutic targets and prognosis. At the same time, the present study focused on Toll-like receptor (TLR) pathways, which exert important immune regulatory functions and have been implicated in tumor progression $(11,12)$.

\section{Materials and methods}

Microarray data. The gene expression profile GSE19804, a comprehensive analysis of the molecular signature of non-smoking patients with non-small cell lung cancer (NSCLC), were obtained from the GEO database. The majority of the tumors were adenocarcinomas (93\%), and $78 \%$ of the samples were in stage I or II. Gene expression profile analysis was performed based on the GPL570 platform (HG-U133-Plus-2; Affymetrix Human Genome U133 Plus 2.0 Array) by Lu et al (13) and then subjected to bioinformatics analysis. There were 120 chips in this dataset. Following quality control by signal strength distribution normalization, correlation analysis and principal component analysis in Agilent Gene-Spring GX v.11.5 (14), the mismatched ones were eliminated and the remaining 31 pairs of cancerous and normal tissues were used for subsequent analysis.

Identification of DEGs. The raw data used for the analysis were pre-processed using the Affy package (version 1.48.0; bioconductor.org/packages/release/bioc/html/affy.html) in $\mathrm{R}$ language (15). Hierarchical clustering analysis was applied to categorize the data into two groups of different expression patterns. Significance analysis by Student's t-test and fold-change (FC) in the expression of genes between each pair of cancerous and normal tissues were jointly used to identify DEGs. Then, the Benjamini and Hochberg method (16) was used to calculate the adjusted P-values [the false discovery rate (FDR)]. The criterion of statistical significance was FDR $<0.05$ and $|\log 2(\mathrm{FC})|>1$.

GO and pathway enrichment analysis of DEGs. The Database for Annotation, Visualization and Integrated Discovery database (DAVID; version 6.8; david.ncifcrf.gov/), an essential tool for systematically extracting biological information from numerous genes (17), was used to perform GO (www.geneontology.org) enrichment and Kyoto Encyclopedia of Genes and Genomes (KEGG; www.genome.jp/) pathway analysis; $\mathrm{P}<0.05$ was considered to indicate a statistically significant difference.

Integration of PPI network and screening of modules. The Search Tool for the Retrieval of Interacting Genes (STRING, version 10.0; string-db.org/) (18), covering 9,643,763 proteins from 2,031 organisms and 932,553,897 interactions, was used to retrieve predicted PPIs. All associations obtained in STRING were provided with a confidence score. Only experimentally validated interactions with a combined score $>0.4$ were selected to construct the PPI network using Cytoscape software (version 3.4.0; www.cytoscape.org/) (19). The Molecular Complex Detection (MCODE) plugin in Cytoscape was utilized to screen the modules of the PPI network, Furthermore, functional and pathway enrichment analyses were performed on the DEGs in the modules. $\mathrm{P}<0.05$ was considered to indicate a statistically significant difference.

\section{Results}

Identification of DEGs. A total of 120 chips were acquired from the GEO datasets. Subsequent to quality control (signal strength distribution normalization, correlation analysis and principal component analysis in Gene-Spring), 62 chips were selected, including data from 31 lung cancer tissues and their matched normal lung tissues. The Pearson's correlation (signal) map (Fig. 1A) and Relative Signal Box Plot map (Fig. 1B) of the pre-treated data presented the performance of normalization. The series from each chip were analyzed separately and the DEGs lists were identified. Information on the expression levels of 54,675 genes was obtained using the GPL570 platform. A total of 1,970 DEGs (cancer tissues vs. normal tissues), including 534 up-regulated and 1,436 down-regulated genes (data not shown), were selected based on the criteria of FDR $<0.05$ and $\mid \log 2$ (FC) $\mid>1$ (Fig. 1C). The statistical metrics for key DEGs was shown in Table I.

Hierarchical clustering analysis of DEGs. Hierarchical clustering analysis was performed for DEGs following the extraction of the expression values. As shown in Fig. 2, the 62 specimens were divided into the lung cancer group and the normal group. The volcano plot demonstrated that compared with normal tissues, there were more downregulated genes than upregulated genes in lung cancer tissues. These results indicated that the DEGs possessed distinct expression patterns in tumors and normal tissues.

GO term enrichment analysis. To investigate the function of the DEGs, GO term enrichment analysis was conducted with the online software DAVID. In general, the DEGs were significantly enriched in biological processes (BP), molecular function (MF) and cellular component (CC) (Table II). In particular, upregulated DEGs were significantly enriched in $\mathrm{BP}$, including 'mitotic cell cycle process', 'mitotic cell cycle', 'cell division', 'chromosome segregation' and 'multicellular organism catabolic process' (Table III). The down-regulated DEGs were also significantly enriched in BP, including 'circulatory system development', 'cardiovascular system development', 'vasculature development', 'blood vessel development' and 'locomotion' (Table III), respectively.

KEGG pathway analysis. KEGG pathway analysis was used to identify pathways for these DEGs. A total of 19 and 58 significantly enriched pathways for up-regulated and down-regulated genes, respectively, were identified. The most significantly enriched pathways associated with lung cancer were 'extracellular matrix (ECM)-receptor interaction', 'malaria', 'complement and coagulation cascades', 'focal adhesion', 'protein digestion and absorption', 'cell adhesion molecules', 'PI3K-Akt signaling pathway', 'Rap1 signaling pathway', 'tight junction' and 'p53 signaling pathway' (Fig. 3 
A

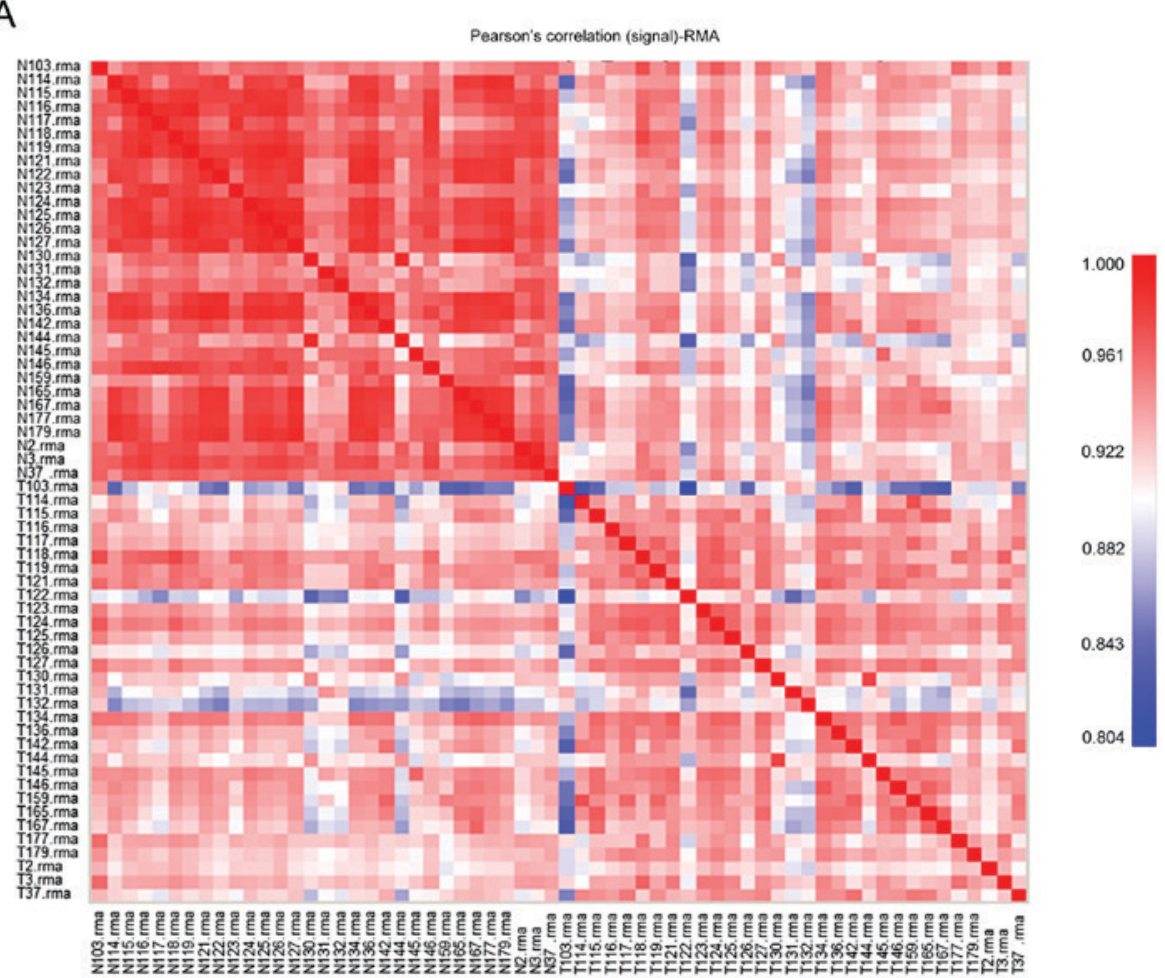

B

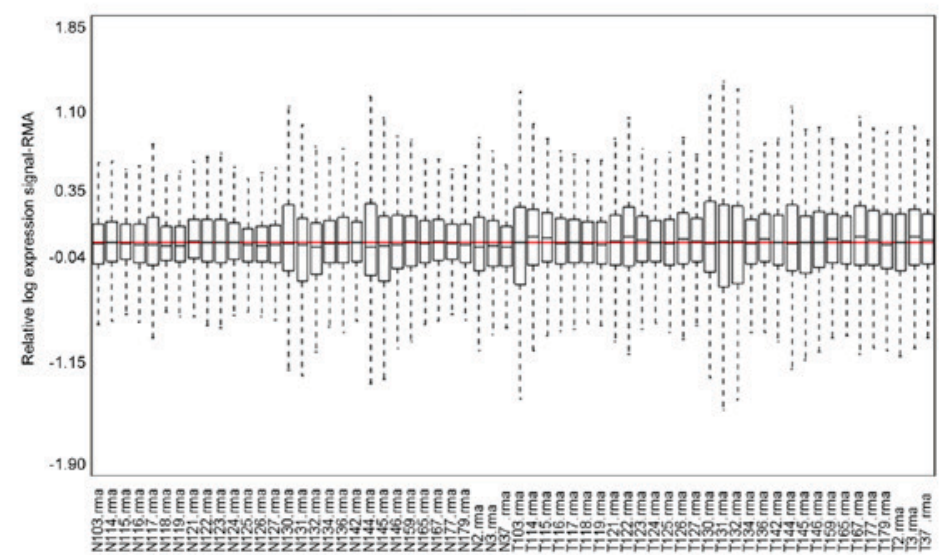

C

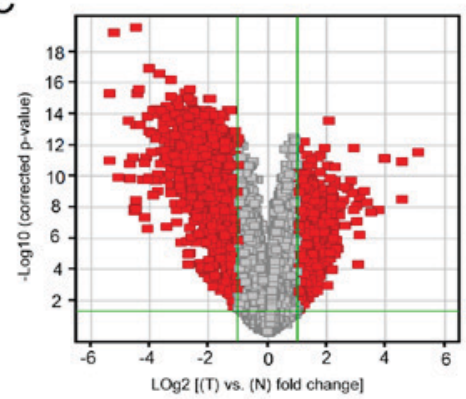

Figure 1. Identification of DEGs. (A) Pearson's correlation (signal) map. The correlation coefficient was close to 1.0 , indicating higher repeatability or similar to distribution. (B) Relative Signal Box Plot map. The red line is the base line; more similar distribution implies higher repeatability of the data. (C) Volcano plot comparing all of the DEGs. The red dots indicate DEGs that were significant at ILog2 (FC)l>1. DEGs, differentially expressed genes; T, lung cancer tissues; $\mathrm{N}$, normal lung tissues.

and Table IV). In addition, the enriched KEGG pathways also included the 'TLR signaling pathway'.

Construction of the PPI network and screening of modules. Based on the predicted interactions of identified DEGs, the PPI network was constructed to identify the most important proteins and biological modules that may serve crucial roles in the development of lung cancer. A total of 1,770 nodes and 10,667 edges were screened from the PPI network (Fig. 4). Each gene was assigned a degree representing the number of neighboring nodes in the network and changes in the proteins/ genes. The top 10 hub nodes with the highest degrees in lung cancer were epidermal growth factor receptor (EGFR), Jun proto-oncogene activator protein (AP)-1 transcription factor subunit (JUN), Fos proto-oncogene AP-1 transcription factor subunit (FOS), interleukin 6 (IL6), MYC proto-oncogene basic helix-loop-helix protein 39 (MYC), matrix metalloproteinase 9 (MMP9), Cyclin dependent kinase 1 (CDK1), Cadherin 1 (CDH1), FYN proto-oncogene Src family tyrosine kinase (FYN) and fibroblast growth factor 2 (FGF2) (Table V). EGFR exhibited the highest node degree of 198 and the betweenness was 0.088 . The high degree of these hub genes indicated that these proteins may serve crucial roles in maintaining the whole protein interaction network. In addition, to explore the significance of these DEGs, the top 3 significant modules were selected, and the functional annotation of the 
Table I. Statistical metrics for key differentially expressed genes.

\begin{tabular}{lllc}
\hline Probe Set ID & \multicolumn{1}{c}{ Gene } & P-value & Fold change (tumor vs. normal) \\
\hline 210081_at & AGER & $1.08 \times 10^{-19}$ & -42.35560 \\
232578_at & CLDN18 & $9.04 \times 10^{-14}$ & -41.43548 \\
204712_at & WIF1 & $1.82 \times 10^{-12}$ & -34.45463 \\
209875_s_at & SPP1 & $2.01 \times 10^{-14}$ & 34.41545 \\
203980_at & FABP4 & $4.45 \times 10^{-17}$ & -27.01589 \\
219230_at & TMEM100 & $4.84 \times 10^{-14}$ & -24.15423 \\
37892_at & COL11A1 & $1.10 \times 10^{-10}$ & 22.98382 \\
209469_at & GPM6A & $5.90 \times 10^{-25}$ & -22.56606 \\
205725_at & SCGB1A1 & $1.21 \times 10^{-10}$ & -22.50023 \\
213317_at & CLIC5 & $1.04 \times 10^{-16}$ & -22.22463
\end{tabular}

AGER, advanced glycosylation end-product specific receptor; CLDN18, claudin 18; WIF1, WNT inhibitory factor 1; SPP1, secreted phosphoprotein 1; FABP4, fatty acid binding protein 4; TMEM100, transmembrane protein 100; COL11A1, collagen type XI $\alpha 1$ chain; GPM6A, glycoprotein M6A; SCGB1A1, secretoglobin family 1A member 1; CLIC5, chloride intracellular channel 5.

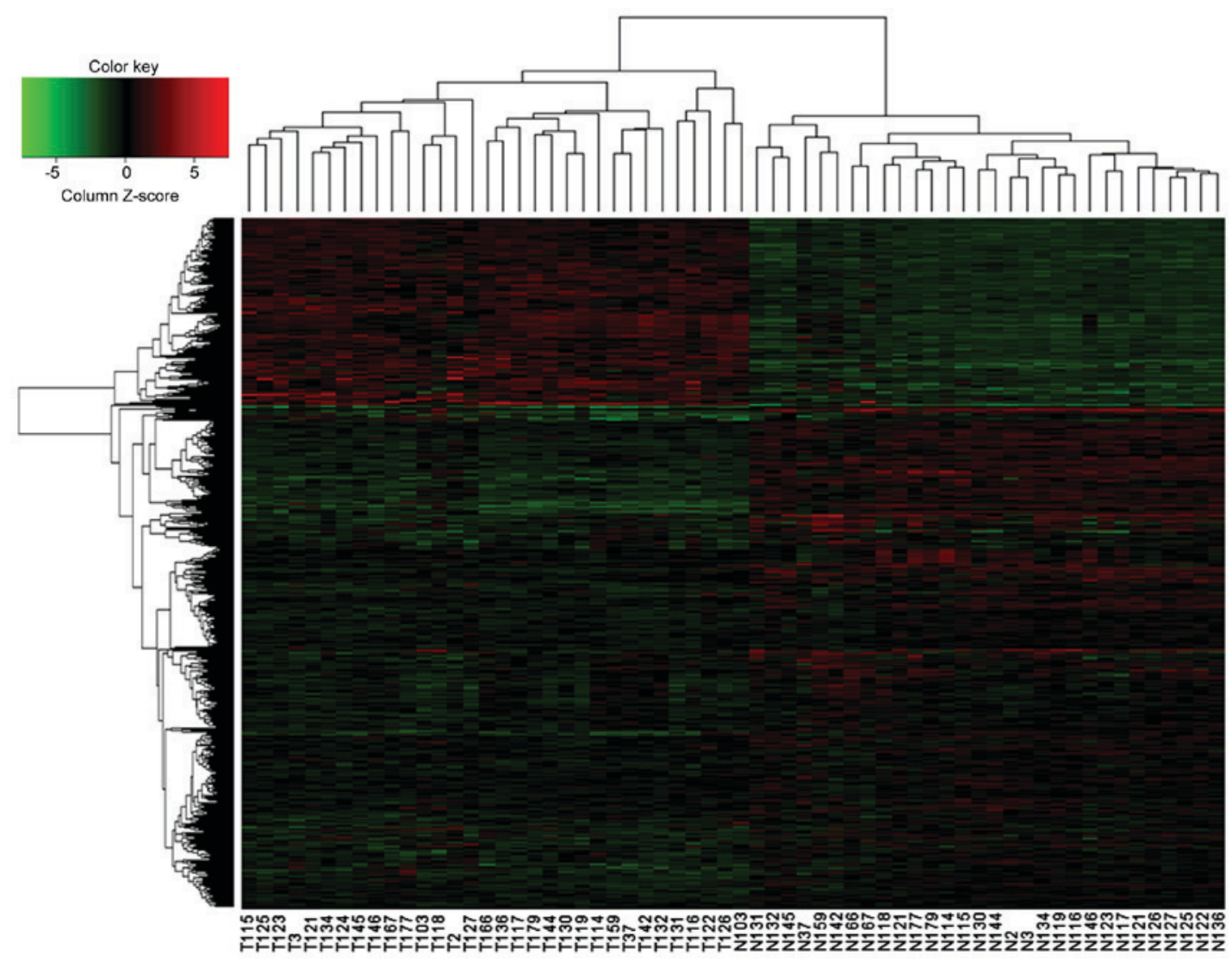

Figure 2. Hierarchical clustering analysis of the 1,970 differentially expressed genes. Red and green indicate higher and lower gene expression, respectively. T, lung cancer tissues; $\mathrm{N}$, normal lung tissues.

genes associated with the modules was analyzed (Fig. 5 and Table VI). The results demonstrated that these modules were associated with the chemokine signaling pathway (Fig. 5A), cell cycle (Fig. 5B) and pathways in cancer (Fig. 5C).

DEGs and pathway analysis of the TLR pathway. Among all of the significantly enriched pathways for DEGs, the TLR signaling pathway was the focus of the present study due to its close association with cancer. There were 12 DEGs primarily involved in this pathway, including secreted phosphoprotein 1, IL6, mitogen-activated protein kinase kinase kinase 8, TLR8, FOS, chemokine ligand 4 (CCL4), TLR4, CCL5, JUN, phosphoinositide-3- kinase regulatory subunit 1 , protein kinase 3 and mitogen-activated protein kinase 13 (Fig. 6 and Table VII). Using the available gene data of this signaling pathway, a network diagram was constructed including a series of receptors, 
Table II. Gene Ontology analysis of differentially expressed genes.

\begin{tabular}{|c|c|c|c|c|}
\hline Gene set name & & Gene counts & $\%$ & P-value \\
\hline GOTERM & GO:0016477-cell migration & 257 & 14.1 & $3.64 \times 10^{-40}$ \\
\hline \multirow[t]{4}{*}{-BP-FAT } & GO:0072358-cardiovascular system development & 222 & 12.2 & $5.09 \times 10^{-40}$ \\
\hline & GO:0072359-circulatory system development & 222 & 12.2 & $5.09 \times 10^{-40}$ \\
\hline & GO:0048870-cell motility & 277 & 15.2 & $5.51 \times 10^{-40}$ \\
\hline & GO:0051674-localization of cell & 277 & 15.2 & $5.51 \times 10^{-40}$ \\
\hline GOTERM & GO:0005102-receptor binding & 222 & 12.2 & $3.09 \times 10^{-14}$ \\
\hline \multirow[t]{4}{*}{-MF-FAT } & GO:0005539-glycosaminoglycan binding & 56 & 3.1 & $4.52 \times 10^{-13}$ \\
\hline & GO:0019838-growth factor binding & 39 & 2.1 & $4.62 \times 10^{-11}$ \\
\hline & GO:0098772-molecular function regulator & 199 & 10.9 & $3.11 \times 10^{-10}$ \\
\hline & GO:0008201-heparin binding & 42 & 2.3 & $1.20 \times 10^{-9}$ \\
\hline GOTERM & GO:0005576-extracellular region & 635 & 34.9 & $4.41 \times 10^{-23}$ \\
\hline \multirow[t]{4}{*}{-CC-FAT } & GO:0044421-extracellular region part & 551 & 30.3 & $1.42 \times 10^{-22}$ \\
\hline & GO:0005615-extracellular space & 253 & 13.9 & $2.95 \times 10^{-20}$ \\
\hline & GO:0005578-proteinaceous extracellular matrix & 97 & 5.3 & $8.71 \times 10^{-20}$ \\
\hline & GO:0031012-extracellular matrix & 123 & 6.8 & $3.43 \times 10^{-19}$ \\
\hline
\end{tabular}

BP, biological processes; FAT, functional annotation tool; MF, molecular function; CC, cellular component.

signaling kinases, transcription factors and cytokines. These genes form a complete signaling pathway to serve an important regulatory role. These results suggested that the TLR pathway could be one of the significant pathways involved in cancer treatment, providing a target for drug development.

\section{Discussion}

Cancer is essentially a genetic disease, and many genetic alterations accumulate during the multistep process of carcinogenesis, which eventually leads to abnormal unrestrained cell growth and malignant phenotype (20). Lung cancer is the most common primary pulmonary malignant tumor in terms of incidence and mortality (21). The total number of lung cancer cases has become a major public health concern in China (rates are 40 per 100,000), and the situation in other parts of world, including Micronesia/Polynesia (rates are $>76.5$ per 100,000) and Eastern Europe (rates are $>61.2$ per 100,000 ) is even worse (3), which is mainly attributable to cigarette smoking (22). Therefore, the early diagnosis and effective treatment of lung cancer is urgently required, which may be achieved via the identification of the DEGs between lung cancer and normal tissues, and by understanding the underlying molecular mechanism. Microarray and high throughput sequencing analysis can screen a large number of genes in the human genome for further functional analysis, and can be widely used to screen biomarkers for early diagnosis and specific therapeutic targets. Therefore, they may aid the diagnosis of lung cancer in the early stages and the development of targeted treatment, thus improving prognosis.

Microarray studies possess great potential to provide novel insights into the pathogenesis of complex diseases (23). The present study systematically applied integrated bioinformatics methods to identify new candidates that serve roles in the progression of lung cancer. The data extracted from the GEO

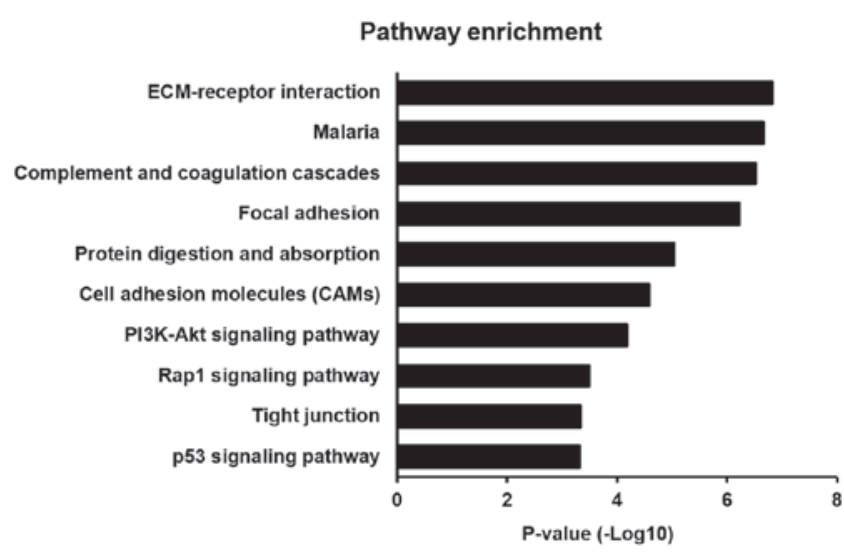

Figure 3. Top 10 most significantly enriched pathways of differentially expressed genes associated with lung cancer as analyzed by Kyoto Encyclopedia of Genes and Genomes pathway analysis. ECM, extracellular matrix; PI3K, phosphoinositide-3-kinase; Akt, protein kinase B; Rap1, Ras-proximate-1.

dataset contained 31 pairs of lung cancer and normal samples. A total of 534 up-regulated and 1,436 down-regulated DEGs in cancerous tissues, when compared with normal samples, were identified using bioinformatics analysis, indicating that the occurrence and development of cancer is closely associated with genetic mutations (24). Then, GO and KEGG pathway analyses were used to investigate the interactions of these DEGs. Finally a PPI network identified specific key genes. The results of the present study may provide potential biomarkers for the diagnosis of lung cancer. For example, it was identified that advanced glycosylation end-product specific receptor (AGER), with a 42-fold decrease in patients with lung cancer, was the most differentially expressed gene in DEG analysis. AGER belongs to the immunoglobulin superfamily and is an oncogenic transmembrane receptor up-regulated in 
Table III. Gene Ontology functional enrichment analyses of differentially expressed genes associated with lung cancer.

A, Up-regulated

\begin{tabular}{|c|c|c|c|c|}
\hline Category & Term/gene function & Gene count & $\%$ & P-value \\
\hline GOTERM_BP_FAT & GO:1903047-mitotic cell cycle process & 62 & 12.5 & $2.47 \times 10^{-12}$ \\
\hline GOTERM_BP_FAT & GO:0000278-mitotic cell cycle & 64 & 12.9 & $9.70 \times 10^{-12}$ \\
\hline GOTERM_BP_FAT & GO:0051301-cell division & 45 & 9.1 & $1.16 \times 10^{-10}$ \\
\hline GOTERM_BP_FAT & GO:0007059-chromosome segregation & 33 & 6.7 & $1.83 \times 10^{-10}$ \\
\hline GOTERM_BP_FAT & $\begin{array}{l}\text { GO:0044243-multicellular organism } \\
\text { catabolic process }\end{array}$ & 16 & 3.2 & $3.58 \times 10^{-10}$ \\
\hline GOTERM_MF_FAT & $\begin{array}{l}\text { GO:0005201-extracellular matrix } \\
\text { structural constituent }\end{array}$ & 12 & 2.4 & $7.34 \times 10^{-6}$ \\
\hline GOTERM_MF_FAT & GO:0042802-identical protein binding & 60 & 12.1 & $3.73 \times 10^{-5}$ \\
\hline GOTERM_MF_FAT & $\begin{array}{l}\text { GO:0008574-ATP-dependent microtubule } \\
\text { motor activity, plus-end-directed }\end{array}$ & 6 & 1.2 & $5.13 \times 10^{-5}$ \\
\hline GOTERM_MF_FAT & $\begin{array}{l}\text { GO: } 1990939-A T P-d e p e n d e n t ~ m i c r o t u b u l e \\
\text { motor activity }\end{array}$ & 6 & 1.2 & $6.96 \times 10^{-5}$ \\
\hline GOTERM_MF_FAT & $\begin{array}{l}\text { GO:0016758-transferase activity, } \\
\text { transferring hexosyl groups }\end{array}$ & 16 & 3.2 & $2.89 \times 10^{-4}$ \\
\hline GOTERM_CC_FAT & GO:0005578-proteinaceous extracellular matrix & 31 & 6.3 & $1.75 \times 10^{-7}$ \\
\hline GOTERM_CC_FAT & GO:0005576-extracellular region & 176 & 35.5 & $5.35 \times 10^{-6}$ \\
\hline GOTERM_CC_FAT & GO:0098643-banded collagen fibril & 6 & 1.2 & $1.26 \times 10^{-5}$ \\
\hline GOTERM_CC_FAT & GO:0005583-fibrillar collagen trimer & 6 & 1.2 & $1.26 \times 10^{-5}$ \\
\hline GOTERM_CC_FAT & $\begin{array}{l}\text { GO:0000779-condensed chromosome, } \\
\text { centromeric region }\end{array}$ & 14 & 1.9 & $1.87 \times 10^{-5}$ \\
\hline
\end{tabular}

B, Down-regulated

\begin{tabular}{|c|c|c|c|c|}
\hline Category & Term/gene function & Gene count & $\%$ & P-value \\
\hline GOTERM_BP_FAT & GO:0072359-circulatory system development & 190 & 14.3 & $3.52 \times 10^{-44}$ \\
\hline GOTERM_BP_FAT & GO:0072358-cardiovascular system development & 190 & 14.3 & $3.52 \times 10^{-44}$ \\
\hline GOTERM_BP_FAT & GO:0001944-vasculature development & 146 & 11.0 & $9.36 \times 10^{-43}$ \\
\hline GOTERM_BP_FAT & GO:0001568-blood vessel development & 137 & 10.3 & $9.96 \times 10^{-40}$ \\
\hline GOTERM_BP_FAT & GO:0040011-locomotion & 242 & 18.3 & $3.86 \times 10^{-38}$ \\
\hline GOTERM_MF_FAT & GO:0005102-receptor binding & 179 & 13.5 & $7.17 \times 10^{-16}$ \\
\hline GOTERM_MF_FAT & GO:0005539-glycosaminoglycan binding & 45 & 3.4 & $6.57 \times 10^{-12}$ \\
\hline GOTERM_MF_FAT & GO:0098772-molecular function regulator & 155 & 11.7 & $2.41 \times 10^{-10}$ \\
\hline GOTERM_MF_FAT & GO:0008201-heparin binding & 36 & 2.7 & $3.61 \times 10^{-10}$ \\
\hline GOTERM_MF_FAT & GO:0003779-actin binding & 62 & 4.7 & $9.32 \times 10^{-10}$ \\
\hline GOTERM_CC_FAT & GO:0044421-extracellular region part & 401 & 30.3 & $6.04 \times 10^{-18}$ \\
\hline GOTERM_CC_FAT & GO:0005576-extracellular region & 459 & 34.6 & $8.97 \times 10^{-18}$ \\
\hline GOTERM_CC_FAT & GO:0005615-extracellular space & 187 & 14.1 & $4.24 \times 10^{-16}$ \\
\hline GOTERM_CC_FAT & GO:0009986-cell surface & 118 & 8.9 & $1.97 \times 10^{-15}$ \\
\hline GOTERM_CC_FAT & GO:0031012-extracellular matrix & 89 & 6.7 & $7.14 \times 10^{-14}$ \\
\hline
\end{tabular}

BP, biological processes; FAT, functional annotation tool; MF, molecular function; CC, cellular component.

various types of human cancers, including squamous cervical cancer (25) and pancreatic tumor (26). However, it has an inhibitory effect on lung cancer development (27). In patients with lung adenocarcinoma, AGER polymorphisms are associated with disease susceptibility and prognosis, and can predict survival $(28,29)$. Therefore, AGER may be effective as a potential marker for lung cancer, however, further studies are required.

GO analysis is helpful for annotating genes and gene products. GO analysis in the present study demonstrated that 
Table IV. Top 10 most overrepresented Kyoto Encyclopedia of Genes and Genomes pathways of differentially expressed genes.

\begin{tabular}{lccc}
\hline Gene set name & Count & $\%$ & P-value \\
\hline hsa04512: ECM-receptor interaction & 28 & 1.5 & $1.52 \times 10^{-7}$ \\
hsa05144: Malaria & 20 & 1.1 & $2.20 \times 10^{-7}$ \\
hsa04610: Complement and coagulation cascades & 24 & 1.3 & $3.03 \times 10^{-7}$ \\
hsa04510: Focal adhesion & 47 & 2.6 & $5.97 \times 10^{-7}$ \\
hsa04974: Protein digestion and absorption & 25 & 1.4 & $9.42 \times 10^{-6}$ \\
hsa04514: Cell adhesion molecules (CAMs) & 33 & 1.8 & $2.59 \times 10^{-5}$ \\
hsa04151: PI3K-Akt signaling pathway & 61 & 3.3 & $6.57 \times 10^{-5}$ \\
hsa04015: Rap1 signaling pathway & 40 & 2.2 & $3.29 \times 10^{-4}$ \\
hsa04530: Tight junction & 29 & 1.6 & $4.68 \times 10^{-4}$ \\
hsa04115: p53 signaling pathway & 18 & 1.0 & $4.77 \times 10^{-4}$
\end{tabular}

ECM, extracellular matrix; PI3K, phosphoinositide-3-kinase; Akt, protein kinase B.

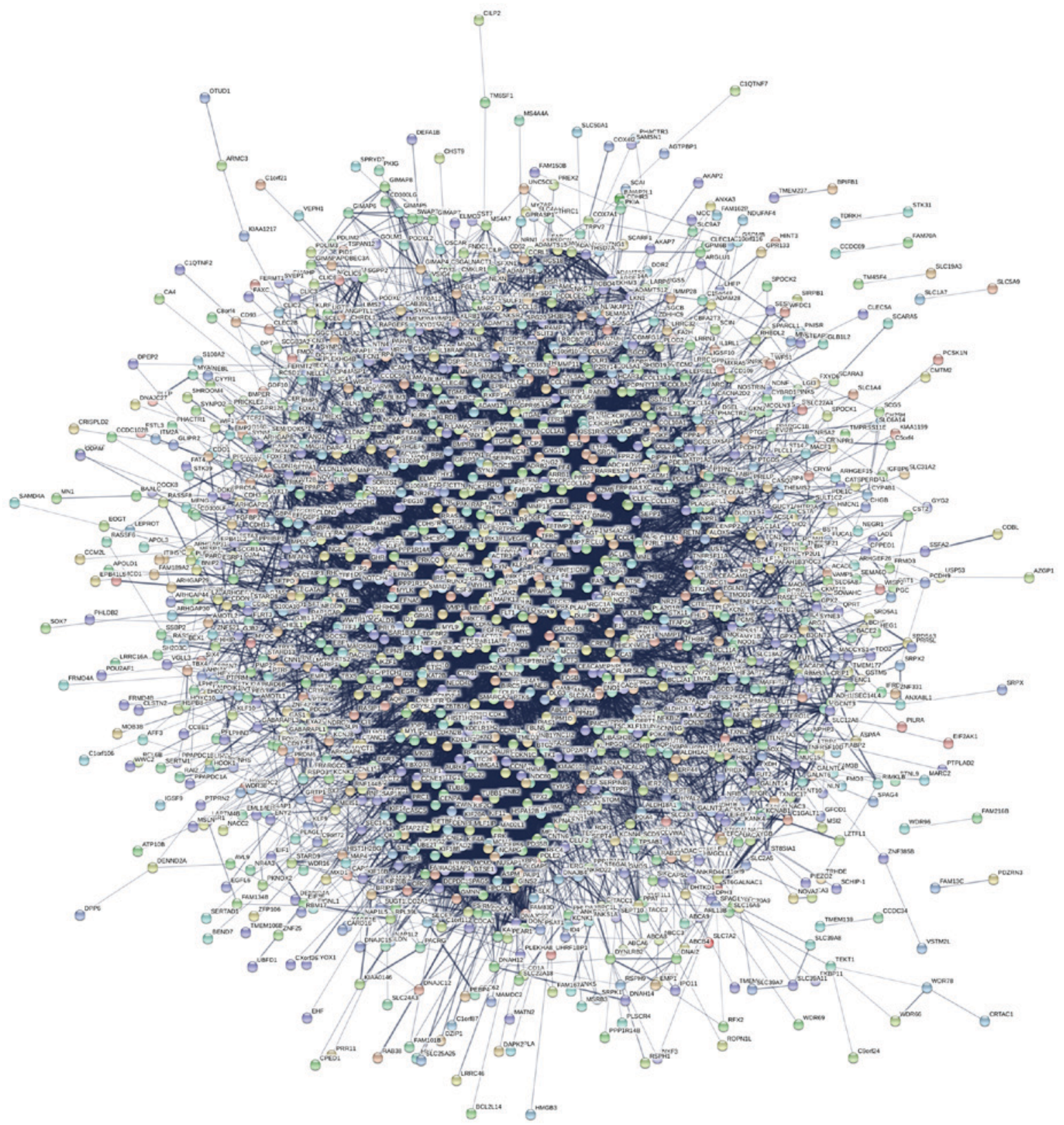

Figure 4. Protein-protein interaction network of differentially expressed genes identified by Search Tool for the Retrieval of Interacting Genes. 
Table V. Top 10 hub nodes with highest degrees of interaction in lung cancer.

\begin{tabular}{lccccc}
\hline Name & Node degree & $\begin{array}{c}\text { Betweenness } \\
\text { centrality }\end{array}$ & $\begin{array}{c}\text { Closeness } \\
\text { centrality }\end{array}$ & Stress centrality & $\begin{array}{r}\text { Clustering } \\
\text { coefficient }\end{array}$ \\
\hline EGFR & 198 & 0.088 & 0.464 & $2,403,514$ & 0.085 \\
JUN & 185 & 0.055 & 0.457 & $1,936,238$ & 0.116 \\
FOS & 164 & 0.038 & 0.447 & $1,365,032$ & 0.120 \\
IL6 & 139 & 0.031 & 0.427 & $1,039,056$ & 0.138 \\
MYC & 136 & 0.030 & 0.441 & $1,089,702$ & 0.147 \\
MMP9 & 135 & 0.028 & 0.433 & $1,011,434$ & 0.148 \\
CDK1 & 121 & 0.032 & 0.427 & 936,828 & 0.105 \\
CDH1 & 119 & 0.035 & 0.426 & $1,062,348$ & 0.111 \\
FYN & 118 & 0.035 & 0.410 & $1,089,798$ & 0.127 \\
FGF2 & 114 & 0.013 & 0.420 & 577,568 & 0.184 \\
\hline
\end{tabular}

A

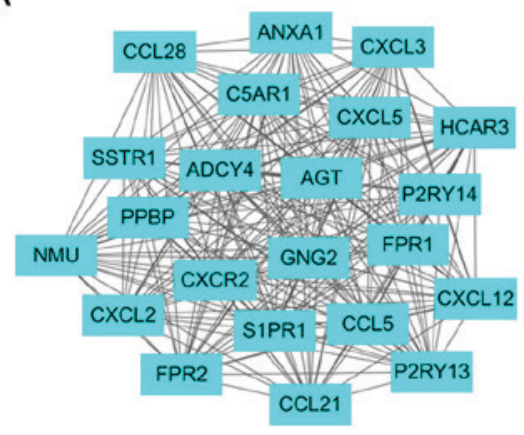

B

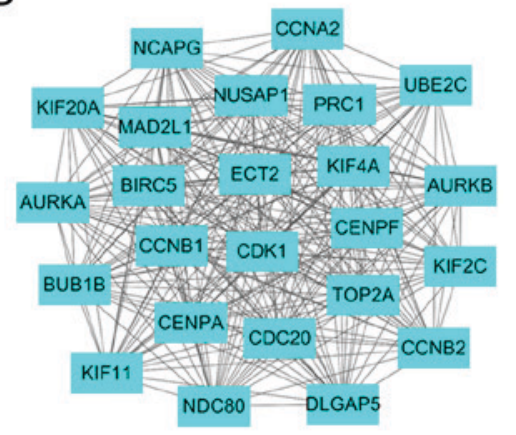

C

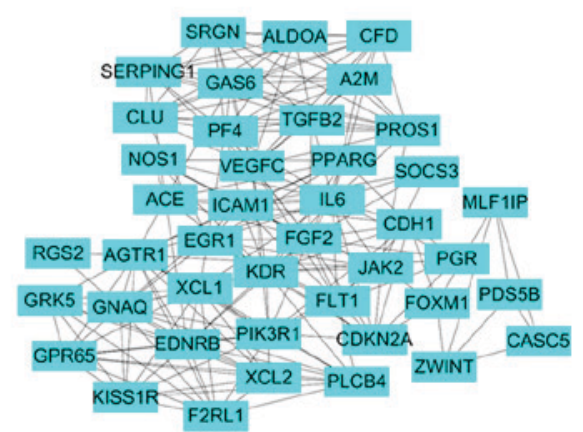

Figure 5. Top 3 modules from the protein-protein interaction network. Nodes and links represent human proteins and protein interactions. (A) The enriched pathways of module 1; (B) The enriched pathways of module 2; (C) The enriched pathways of module 3.

up-regulated DEGs were mainly involved in the cell cycle and material metabolism, including 'mitotic cell cycle', 'cell division' and 'multicellular organism catabolic' processes, which mainly refer to cellular processes. However, the down-regulated DEGs were mainly involved in organ systems, including the circulatory system, cardiovascular system and vasculature development, all of which are associated with systemic processes. This is consistent with the knowledge that the defective functioning of cell biological processes (30) and the state of the body system status are important causes of tumor development and progression. Also it indicated that cellular activity was enhanced and system function was weakened. Therefore, monitoring the expression of these DEGs may aid the discovery of mechanisms for tumorigenesis and tumor progression. It is known that signal transduction in cancer cells differs substantially from normal cells (31). The KEGG pathway database contains information on systematic analysis of gene functions, linking genomics with functional information. Enrichment analysis identified important KEGG pathways associated with lung cancer, including 'ECM-receptor interaction', 'malaria', 'complement and coagulation cascades', 'focal adhesion', and 'protein digestion and absorption'. Pathway disturbance in 'ECM-receptor interaction', 'complement and coagulation cascades' and 'adhesive attraction' (32-34) have been highly noted in lung cancer. The present study identified that the ECM-tumor cell interactions may activate intracellular signaling pathways, which is responsible for tumor cell invasion and metastasis $(35,36)$.

The present study focused on the TLR pathway among the enriched pathways that are expressed in a wide variety of cancer cells and immune cells (37). This pathway is centrally involved in the initiation of innate immunity and induction of adaptive immune responses (38), which is useful for maintaining organism integrity and is markedly involved in cancer progression, development and defense (39). However, the activation of TLRs in tumor cells induces the synthesis of pro-inflammatory factors and immunosuppressive molecules, which can enhance the resistance of tumor cells to cytotoxic lymphocyte attack and lead to immune evasion (40). Cancer cells can avoid immune surveillance as TLRs trigger cells to release a number of biological factors, including IL6, vascular endothelial growth factor and MMP (41). Notably, these factors were included in the DEGs between lung cancer tissues and normal tissues. Therefore, it was suggested that targeting tumor TLR signaling pathways may provide promising therapeutic methods (40).

A PPI network was constructed with DEGs, which revealed that the top 10 hub genes with highest degrees were EGFR, JUN, FOS, IL6, MYC, MMP9, CDK1, CDH1, FYN and FGF2. EGFR was at the core of the PPI network and exhibited 
Table VI. Top 3 modules from the protein-protein interaction network.

\begin{tabular}{|c|c|c|c|c|}
\hline Modules & Term & P-value & $\begin{array}{l}\text { False } \\
\text { discovery rate }\end{array}$ & Genes \\
\hline \multirow[t]{3}{*}{1} & $\begin{array}{l}\text { Chemokine signaling } \\
\text { pathway }\end{array}$ & $6.11 \times 10^{-12}$ & $6.23 \times 10^{-9}$ & $\begin{array}{l}\text { ADCY4, CXCL5, PPBP, CCL21, CXCL3, } \\
\text { CXCL2, CXCR2, GNG2, CCL5, CXCL12, } \\
\text { CCL28 }\end{array}$ \\
\hline & $\begin{array}{l}\text { Neuroactive ligand- } \\
\text { receptor interaction }\end{array}$ & $5.62 \times 10^{-5}$ & 0.057248 & $\begin{array}{l}\text { P2RY13, S1PR1, C5AR1, SSTR1, P2RY14, } \\
\text { FPR1, FPR2 }\end{array}$ \\
\hline & $\begin{array}{l}\text { Cytokine-cytokine } \\
\text { receptor interaction }\end{array}$ & $2.51 \times 10^{-4}$ & 0.255655 & $\begin{array}{l}\text { PPBP, CCL21, CXCR2, CCL5, CXCL12, } \\
\text { CCL28 }\end{array}$ \\
\hline \multirow[t]{3}{*}{2} & Cell cycle & $2.53 \times 10^{-9}$ & $1.80 \times 10^{-6}$ & $\begin{array}{l}\text { CCNB1, CDK1, CCNB2, MAD2L1, BUB1B, } \\
\text { CDC20, CCNA2 }\end{array}$ \\
\hline & $\begin{array}{l}\text { Progesterone-mediated } \\
\text { oocyte maturation }\end{array}$ & $2.90 \times 10^{-6}$ & 0.002056 & CCNB1, CDK1, CCNB2, MAD2L1, CCNA2 \\
\hline & Oocyte meiosis & $2.99 \times 10^{-4}$ & 0.212472 & CDK1, MAD2L1, CDC20, AURKA \\
\hline \multirow[t]{3}{*}{3} & Pathways in cancer & $7.30 \times 10^{-7}$ & $8.72 \times 10^{-4}$ & $\begin{array}{l}\text { AGTR1, EDNRB, VEGFC, IL6, CDKN2A, } \\
\text { PLCB4, GNAQ, PPARG, CDH1, FGF2, } \\
\text { PIK3R1, TGFB2 }\end{array}$ \\
\hline & $\begin{array}{l}\text { Chagas disease } \\
\text { (American } \\
\text { trypanosomiasis) }\end{array}$ & $9.65 \times 10^{-6}$ & 0.011527 & ACE, IL6, PLCB4, GNAQ, PIK3R1, TGFB2 \\
\hline & African trypanosomiasis & $2.00 \times 10^{-5}$ & 0.023928 & ICAM1, IL6, PLCB4, GNAQ, F2RL1 \\
\hline
\end{tabular}

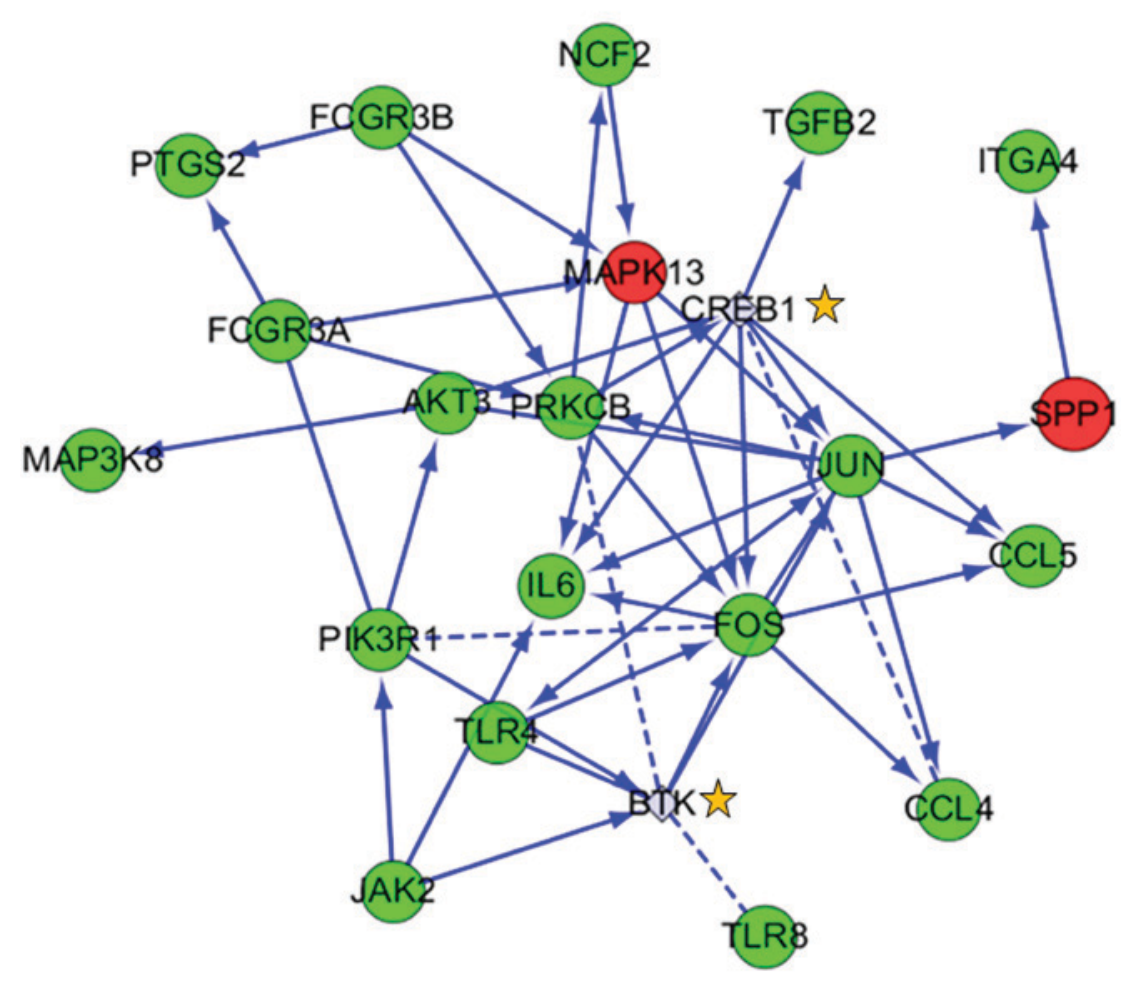

Figure 6. Gene network associated with Toll-like receptor pathways. Red and green dots represent up- and downregulated differentially expressed genes, respectively. Grey diamonds marked with yellow stars represent the associated genes, which were added by the system. The dot size represents the difference of one gene between lung cancer tissues and normal tissues.

the highest degree with a connectivity of 198, suggesting a role for EGFR as a potential marker for lung cancer. EGFR is a transmembrane receptor tyrosine kinase and is frequently observed in lung cancer patients with poor differentiation 
Table VII. Main genes associated with Toll-like receptors pathways.

\begin{tabular}{lllr}
\hline Gene ID & Gene & P-value & Fold change \\
\hline 6696 & SPP1 & $2.01 \times 10^{-14}$ & 34.41545 \\
3569 & IL6 & $1.05 \times 10^{-12}$ & -8.05396 \\
1326 & MAP3K8 & $5.29 \times 10^{-15}$ & -4.17527 \\
51311 & TLR8 & $5.01 \times 10^{-10}$ & -3.84351 \\
2353 & FOS & $5.20 \times 10^{-12}$ & -3.81020 \\
6351 & CCL4 & $5.31 \times 10^{-9}$ & -2.77206 \\
7099 & TLR4 & $3.40 \times 10^{-7}$ & -2.50968 \\
6352 & CCL5 & $1.51 \times 10^{-6}$ & -2.42054 \\
3725 & JUN & $1.05 \times 10^{-8}$ & -2.36435 \\
5295 & PIK3R1 & $8.86 \times 10^{-10}$ & -2.26284 \\
10000 & AKT3 & $9.98 \times 10^{-11}$ & -2.22438 \\
5603 & MAPK13 & $7.48 \times 10^{-11}$ & 2.14546 \\
\hline
\end{tabular}

and poor prognosis (42). It can lead to signal transduction including cell differentiation, proliferation and apoptosis through phosphorylating other proteins, and has been reported to be overexpressed in patients with NSCLC (43). Activating mutations in the EGFR gene are considered to be favorable prognostic markers (44) and have become a novel personalized treatment target for patients with NSCLC (45). However, the role of EGFR as a marker of lung cancer diagnosis and treatment is unclear and requires further investigation. JUN and FOS are the predominant components of AP-1, which serves a critical role in the transcriptional regulation of genes involved in cell survival, proliferation, migration and transformation (46). These two proteins are overexpressed in the development of various carcinomas, including pulmonary malignancies (47). The IL6 gene is co-expressed with a number of oncogenic genes, and IL6 is produced and secreted by immune and tumor cells (48). It is involved in different physiologic and pathophysiologic processes such as promoting tumorigenesis and modifying various tumor behaviors, including apoptosis, migration, proliferation, angiogenesis and metabolism $(49,50)$. Elevated levels of serum IL6 are associated with poor prognosis in the majority of malignancies (51). The MYC gene is an oncogene with a high frequency of amplification in lung cancer (52) and its protein has important roles in cell proliferation and differentiation. It is closely associated with tumor occurrence, progression and prognosis in different types of tumors (53). The other 5 hub genes also serve important roles in cell migration, proliferation, differentiation, apoptosis and cell cycle process (54-56), affecting the development of cancer. MMP9 degrades and restores the ECM, and its overexpression not only promotes NSCLC metastasis directly but can also facilitate metastatic spread (57). CDK1 is expressed in high levels in tumor tissues, and is associated with poor prognosis and shorter survival time in patients with NSCLC (58). The CDH1 gene encodes a tumor suppressor protein and its mutation or deletion results in the promotion of cancer invasion and metastasis (55) in addition to poor prognosis (59). FYN contributes to the progression of cancer by regulating cell cycle, differentiation, adhesion, motility and survival of cells (56). FGF2 has been shown to be activated in lung cancer (60) and is involved in angiogenesis by initiating a signal transduction cascade that promotes cell proliferation, motility and angiogenesis (61). Therefore, all the hub genes may possess key roles in lung cancer and could interact with each other. They may be used as potential effective candidates for early diagnosis or prognosis.

The PPI module contained 1,770 nodes and 10,667 edges, and the top 3 modules extracted were 'chemokine signaling pathway', 'cell cycle' and 'pathways in cancer', all of which are associated with lung cancer. The chemokine signaling pathway contains a number of chemokine proteins, including chemokine (C-X-C motif) ligand (CXCL)-2, CXCL3 and CCL18, which are differentially expressed in lung cancer. As chemokine gradients direct cell migration towards the site of inflammation (62), they are key in immune system functioning, and are important for the removal of pathogens, inflammation, cell and organ development, wound repair, occurrence of tumors and metastasis, and transplantation immune rejection (63-65). Cell cycle disorders and overgrowth of cells are common biological characteristics of tumors, leading to increased cell proliferation and decreased apoptosis (66). It should be noted that the cell cycle is a tightly regulated process and is frequently aberrant in lung cancer (67). The relevant inhibitors of the cell cycle have emerged as novel drugs for the treatment of lung cancer by suppressing the unrestricted cell division and growth of lung cancer cells (67). Finally, pathways in cancer are of similar significance in cancer cell proliferation, apoptosis, metastasis, angiogenesis and survival.

However, there were still certain limitations in the present study. First, although a set number of genes were revealed to be potential markers for lung cancer, further experiments are still required to evaluate the roles of these genes as novel biomarkers. Secondly, the gene expression profile was composed of $93 \%$ adenocarcinomas and $7 \%$ squamous cancer, since there is great genetic heterogeneity between adenocarcinoma and squamous cancer. Furthermore, the variation trend of JUN, FOS, IL6, MYC and FGF2 in this study is inconsistent with that reported in previous studies $(46,47)$; this may be due to the limited numbers of chips. Therefore, further data for lung cancer are required to validate the results of the present 
study. Thirdly, due to the limitation of the dataset used, it was not possible to construct a miRNA-target gene regulatory network or transcription factor-target gene regulatory network. Finally, survival analysis could not be performed based on the data presented in the present study.

In conclusion, the present study provided a comprehensive bioinformatics analysis of DEGs in lung cancer. Analysis of these altered genes provided information regarding the molecular mechanisms of lung cancer and significant biomarkers or targets for the diagnosis and treatment of lung cancer. However, further molecular biological experiments are required to confirm the function of the DEGs and pathways in different types of lung cancer.

\section{Acknowledgements}

Not applicable.

\section{Funding}

The present study was supported by the Funds of National Natural Science Foundation of China (grant nos. 81473388 and 8274144).

\section{Availability of data and materials}

The datasets used and/or analyzed in the current study are available from the corresponding authors on reasonable request.

\section{Authors' contributions}

TL and YB conceived and designed the study. ZL and XZ analyzed the microarray datasets and interpreted the results. SY and HT downloaded the gene expression profile from the Gene Expression Omnibus. TL wrote and edited the manuscript. All authors read and approved the final manuscript.

\section{Ethics approval and consent to participate}

Not applicable.

\section{Patient consent for publication}

Not applicable.

\section{Competing interests}

The authors declare that they have no competing interests.

\section{References}

1. Cancer Genome Atlas Research Network: Comprehensive molecular profiling of lung adenocarcinoma. Nature 511: 543-550, 2014.

2. Cohen AJ, Brauer M, Burnett R, Anderson HR, Frostad J, Estep K, Balakrishnan K, Brunekreef B, Dandona L, Dandona R, et al: Estimates and 25-year trends of the global burden of disease attributable to ambient air pollution: An analysis of data from the Global Burden of Diseases Study 2015. Lancet 389: 1907-1918, 2017.

3. Bray F, Ferlay J, Soerjomataram I, Siegel RL, Torre LA and Jemal A: Global cancer statistics 2018: GLOBOCAN estimates of incidence and mortality worldwide for 36 cancers in 185 countries. CA Cancer J Clin 68: 394-424, 2018.
4. Wang LQ, Zhao LH and Qiao YZ: Identification of potential therapeutic targets for lung cancer by bioinformatics analysis. Mol Med Rep 13: 1975-1982, 2016.

5. Aran V, Victorino AP, Thuler LC and Ferreira CG: Colorectal cancer: Epidemiology, disease mechanisms and interventions to reduce onset and mortality. Clin Colorectal Cancer 15: 195-203, 2016.

6. Zhang M, Gao C, Yang Y, Li G, Dong J, Ai Y, Ma Q and Li W: MiR-424 promotes non-small cell lung cancer progression and metastasis through regulating the tumor suppressor gene TNFAIP1. Cell Physiol Biochem 42: 211-221, 2017.

7. Singleton KR, Hinz TK, Kleczko EK, Marek LA, Kwak J, Harp T, Kim J, Tan AC and Heasley LE: Kinome RNAi screens reveal synergistic targeting of MTOR and FGFR1 pathways for treatment of lung cancer and HNSCC. Cancer Res 75: 4398-4406, 2015.

8. Liang B, Li C and Zhao J: Identification of key pathways and genes in colorectal cancer using bioinformatics analysis. Med Oncol 33: 111, 2016.

9. Descotes F, Dessen P, Bringuier PP, Decaussin M, Martin PM, Adams M, Villers A, Lechevallier E, Rebillard X, Rodriguez-Lafrasse C, et al: Microarray gene expression profiling and analysis of bladder cancer supports the sub-classification of T1 tumours into T1a and T1b stages. BJU Int 113: 333-342, 2014.

10. Sakashita H, Inoue H, Akamine S, Ishida T, Inase N, Shirao K, Mori M and Mimori K: Identification of the NEDD4L gene as a prognostic marker by integrated microarray analysis of copy number and gene expression profiling in non-small cell lung cancer. Ann Surg Oncol 20 (Suppl 3): S590-S598, 2013.

11. Yu L, Wang L and Chen S: Dual character of Toll-like receptor signaling: Pro-tumorigenic effects and anti-tumor functions. Biochim Biophys Acta 1835: 144-154, 2013.

12. Pinto A, Morello S and Sorrentino R: Lung cancer and Toll-like receptors. Cancer Immunol Immunother 60: 1211-1220, 2011.

13. Lu TP, Tsai MH, Lee JM, Hsu CP, Chen PC, Lin CW, Shih JY, Yang PC, Hsiao CK, Lai LC and Chuang EY: Identification of a novel biomarker, SEMA5A, for non-small cell lung carcinoma in nonsmoking women. Cancer Epidemiol Biomarkers Prev 19: 2590-2597, 2010

14. Hu WP, Zeng YY, Zuo YH and Zhang J: Identification of novel candidate genes involved in the progression of emphysema by bioinformatic methods. Int J Chron Obstruct Pulmon Dis 13: 3733-3747, 2018.

15. Gautier L, Cope L, Bolstad BM and Irizarry RA: Affy-analysis of Affymetrix GeneChip data at the probe level. Bioinformatics 20 : 307-315, 2004.

16. Hardcastle TJ: Generalised empirical Bayesian methods for discovery of differential data in high-throughput biology. Bioinformatics 32: 195-202, 2016.

17. Huang DW, Sherman BT, Tan Q, Kir J, Liu D, Bryant D, Guo Y, Stephens R, Baseler MW, Lane HC and Lempicki RA: DAVID Bioinformatics Resources: Expanded annotation database and novel algorithms to better extract biology from large gene lists. Nucleic Acids Res 35: W169-W175, 2007.

18. Franceschini A, Szklarczyk D, Frankild S, Kuhn M, Simonovic M, Roth A, Lin J, Minguez P, Bork P, von Mering C and Jensen LJ: STRING v9.1: Protein-protein interaction networks, with increased coverage and integration. Nucleic Acids Res 41: D808-D815, 2013.

19. Bader GD and Hogue CW: An automated method for finding molecular complexes in large protein interaction networks. BMC Bioinformatics 4: 2, 2003.

20. Carter SL, Eklund AC, Kohane IS, Harris LN and Szallasi Z: A signature of chromosomal instability inferred from gene expression profiles predicts clinical outcome in multiple human cancers. Nat Genet 38: 1043-1048, 2006.

21. Dela Cruz CS, Tanoue LT and Matthay RA: Lung cancer: Epidemiology, etiology, and prevention. Clin Chest Med 32: 605-644, 2011.

22. Wang GZ, Cheng X, Li XC, Liu YQ, Wang XQ, Shi X, Wang ZY, Guo YQ, Wen ZS, Huang YC and Zhou GB: Tobacco smoke induces production of chemokine CCL20 to promote lung cancer. Cancer Lett 363: 60-70, 2015.

23. Masuda K, Kuwano Y, Nishida K and Rokutan K: Application of DNA microarray technology to gerontological studies. Methods Mol Biol 1048: 285-308, 2013.

24. Chew SK, Lu D, Campos LS, Scott KL, Saci A, Wang J, Collinson A, Raine K, Hinton J, Teague JW, et al: Polygenic in vivo validation of cancer mutations using transposons. Genome Biol 15: 455, 2014. 
25. Zhu X, Zhou L, Li R, Shen Q, Cheng H, Shen Z and Zhu H: AGER promotes proliferation and migration in cervical cancer. Biosci Rep 38: BSR20171329, 2018.

26. Kang R,Tang D,LotzeMTandZehHJIII: AGER/RAGE-mediated autophagy promotes pancreatic tumorigenesis and bioenergetics through the IL6-pSTAT3 pathway. Autophagy 8: 989-991, 2012.

27. Pan Z, Liu L, Nie W, Miggin S, Qiu F, Cao Y, Chen J, Yang B, Zhou Y, Lu J and Yang L: Long non-coding RNA AGER-1 functionally upregulates the innate immunity gene AGER and approximates its anti-tumor effect in lung cancer. Mol Carcinog 57: 305-318, 2018.

28. Yamaguchi K, Iwamoto H, Sakamoto S, Horimasu Y, Masuda T, Miyamoto S, Nakashima T, Ohshimo S, Fujitaka K, Hamada H and Hattori N: AGER rs2070600 polymorphism elevates neutrophil-lymphocyte ratio and mortality in metastatic lung adenocarcinoma. Oncotarget 8: 94382-94392, 2017.

29. Wang X, Cui E, Zeng H, Hua F, Wang B, Mao W and Feng X: RAGE genetic polymorphisms are associated with risk, chemotherapy response and prognosis in patients with advanced NSCLC. PLoS One 7: e43734, 2012.

30. Evan GI and Vousden KH: Proliferation, cell cycle and apoptosis in cancer. Nature 411: 342-348, 2001.

31. Kolch W, Halasz M, Granovskaya M and Kholodenko BN: The dynamic control of signal transduction networks in cancer cells. Nat Rev Cancer 15: 515-527, 2015.

32. Lv M and Wang L: Comprehensive analysis of genes, pathways, and TFs in nonsmoking Taiwan females with lung cancer. Exp Lung Res 41: 74-83, 2015.

33. Ajona D, Pajares MJ, Corrales L, Perez-Gracia JL, Agorreta J, Lozano MD, Torre W, Massion PP, de-Torres JP, Jantus-Lewintre E, et al: Investigation of complement activation product $\mathrm{c} 4 \mathrm{~d}$ as a diagnostic and prognostic biomarker for lung cancer. J Natl Cancer Inst 105: 1385-1393, 2013.

34. Fonseca FL, Azzalis LA, Feder D, Nogoceke E, Junqueira VB, Valenti VE and de Abreu LC: Adhesion molecules affected by treatment of lung cancer cells with epidermal growth factor. Lung 189: 383-389, 2011.

35. Wang $\mathrm{H}$, Zhu Y, Zhao M, Wu C, Zhang P, Tang L, Zhang H, Chen X, Yang Y and Liu G: miRNA-29c suppresses lung cancer cell adhesion to extracellular matrix and metastasis by targeting integrin $\beta 1$ and matrix metalloproteinase 2 (MMP2). PLoS One 8: e70192, 2013.

36. Ungewiss C, Rizvi ZH, Roybal JD, Peng DH, Gold KA, Shin DH, Creighton CJ and Gibbons DL: The microRNA-200/Zeb1 axis regulates ECM-dependent $\beta 1$-integrin/FAK signaling, cancer cell invasion and metastasis through CRKL. Sci Rep 6: 18652, 2016.

37. Rakoff-Nahoum S and Medzhitov R: Toll-like receptors and cancer. Nat Rev Cancer 9: 57-63, 2009.

38. Kawai T and Akira S: The role of pattern-recognition receptors in innate immunity: Update on Toll-like receptors. Nat Immunol 11: 373-384, 2010.

39. Chen R, Alvero AB, Silasi DA, Steffensen KD and Mor G: Cancers take their Toll-the function and regulation of Toll-like receptors in cancer cells. Oncogene 27: 225-233, 2008.

40. Huang B, Zhao J, Unkeless JC, Feng ZH and Xiong H: TLR signaling by tumor and immune cells: A double-edged sword. Oncogene 27: 218-224, 2008 .

41. Li D, Jin Y, Sun Y, Lei J and Liu C: Knockdown of toll-like receptor 4 inhibits human NSCLC cancer cell growth and inflammatory cytokine secretion in vitro and in vivo. Int J Oncol 45 813-821, 2014

42. Jo U, Park KH, Whang YM, Sung JS, Won NH, Park JK and Kim YH: EGFR endocytosis is a novel therapeutic target in lung cancer with wild-type EGFR. Oncotarget 5: 1265-1278, 2014.

43. Sequist LV and Lynch TJ: EGFR tyrosine kinase inhibitors in lung cancer: An evolving story. Annu Rev Med 59: 429-442, 2008 .

44. Fang $\mathrm{S}$ and Wang Z: EGFR mutations as a prognostic and predictive marker in non-small-cell lung cancer. Drug Des Devel Ther 8: 1595-1611, 2014.

45. Moschini I, Dell'Anna C, Losardo PL, Bordi P, D'Abbiero N and Tiseo M: Radiotherapy of non-small-cell lung cancer in the era of EGFR gene mutations and EGF receptor tyrosine kinase inhibitors. Future Oncol 11: 2329-2342, 2015

46. Li Y, Yang Z, Li W, Xu S, Wang T, Wang T, Niu M, Zhang S, Jia L and Li S: TOPK promotes lung cancer resistance to EGFR tyrosine kinase inhibitors by phosphorylating and activating c-Jun. Oncotarget 7: 6748-6764, 2016.
47. Durchdewald M, Angel P and Hess J: The transcription factor Fos: A Janus-type regulator in health and disease. Histol Histopathol 24: 1451-1461, 2009.

48. Ataie-Kachoie P, Pourgholami MH and Morris DL: Inhibition of the IL-6 signaling pathway: A strategy to combat chronic inflammatory diseases and cancer. Cytokine Growth Factor Rev 24: 163-173, 2013.

49. Kumari N, Dwarakanath BS, Das A and Bhatt AN: Role of interleukin-6 in cancer progression and therapeutic resistance. Tumour Biol 37: 11553-11572, 2016.

50. Nilsson MB, Langley RR and Fidler IJ: Interleukin-6, secreted by human ovarian carcinoma cells, is a potent proangiogenic cytokine. Cancer Res 65: 10794-10800, 2005.

51. Heikkilä K, Ebrahim S and Lawlor DA: Systematic review of the association between circulating interleukin-6 (IL-6) and cancer. Eur J Cancer 44: 937-945, 2008.

52. Tai MC, Kajino T, Nakatochi M, Arima C, Shimada Y, Suzuki M, Miyoshi H, Yatabe Y, Yanagisawa K and Takahashi T: miR-342-3p regulates MYC transcriptional activity via direct repression of E2F1 in human lung cancer. Carcinogenesis 36: 1464-1473, 2015

53. Gabay M, Li Y and Felsher DW: MYC activation is a hallmark of cancer initiation and maintenance. Cold Spring Harb Perspect Med 4: a014241, 2014

54. Sica A, Allavena P and Mantovani A: Cancer related inflammation: The macrophage connection. Cancer Lett 267: 204-215, 2008.

55. Yu Q, Guo Q, Chen L and Liu S: Clinicopathological significance and potential drug targeting of $\mathrm{CDH} 1$ in lung cancer: $\mathrm{A}$ meta-analysis and literature review. Drug Des Devel Ther 9: 2171-2178, 2015.

56. Thomas SM and Brugge JS: Cellular functions regulated by Src family kinases. Annu Rev Cell Dev Biol 13: 513-609, 1997.

57. Zhen Y, Liu J, Huang Y, Wang Y, Li W and Wu J: miR-133b inhibits cell growth, migration, and invasion by targeting MMP9 in non-small cell lung cancer. Oncol Res 25: 1109-1116, 2017

58. Zhang C, Elkahloun AG, Robertson M, Gills JJ, Tsurutani J, Shih JH, Fukuoka J, Hollander MC, Harris CC, Travis WD, et al: Loss of cytoplasmic CDK1 predicts poor survival in human lung cancer and confers chemotherapeutic resistance. PLoS One 6: e23849, 2011

59. Liu X, Su L and Liu X: Loss of CDH1 up-regulates epidermal growth factor receptor via phosphorylation of YBX1 in non-small cell lung cancer cells. FEBS Lett 587: 3995-4000, 2013.

60. Behrens C, Lin HY, Lee JJ, Raso MG, Hong WK, Wistuba II and Lotan R: Immunohistochemical expression of basic fibroblast growth factor and fibroblast growth factor receptors 1 and 2 in the pathogenesis of lung cancer. Clin Cancer Res 14: 6014-6022, 2008.

61. Ribatti D, Vacca A, Rusnati M and Presta M: The discovery of basic fibroblast growth factor/fibroblast growth factor-2 and its role in haematological malignancies. Cytokine Growth Factor Rev 18: 327-334, 2007.

62. Zhang L, Yu M, Deng J, Lv X, Liu J, Xiao Y, Yang W, Zhang Y and Li C: Chemokine signaling pathway involved in CCL2 expression in patients with rheumatoid arthritis. Yonsei Med J 56: 1134-1142, 2015.

63. González-Motos V, Jürgens C, Ritter B, Kropp KA, Durán V, Larsen O, Binz A, Ouwendijk WJD, Lenac Rovis T, Jonjic S, et al: Varicella zoster virus glycoprotein $\mathrm{C}$ increases chemokine-mediated leukocyte migration. PLoS Pathog 13: e1006346, 2017.

64. Griffith JW, Sokol CL and Luster AD: Chemokines and chemokine receptors: Positioning cells for host defense and immunity. Annu Rev Immunol 32: 659-702, 2014.

65. Franciszkiewicz K, Boissonnas A, Boutet $\mathrm{M}$, Combadière $\mathrm{C}$ and Mami-Chouaib F: Role of chemokines and chemokine receptors in shaping the effector phase of the antitumor immunonse. Cancer Res 72: 6325-6332, 2012.

66. Hsiao CJ, Hsiao G, Chen WL, Wang SW, Chiang CP, Liu LY, Guh JH, Lee TH and Chung CL: Cephalochromin induces G0/G1 cell cycle arrest and apoptosis in A549 human non-small-cell lung cancer cells by inflicting mitochondrial disruption. J Nat Prod 77: 758-765, 2014.

67. Shcherba M, Liang Y, Fernandes D, Perez-Soler R and Cheng H: Cell cycle inhibitors for the treatment of NSCLC. Expert Opin Pharmacother 15: 991-1004, 2014.

This work is licensed under a Creative Commons Attribution-NonCommercial-NoDerivatives 4.0 International (CC BY-NC-ND 4.0) License. 